\title{
RIGID SUBSETS IN EUCLIDEAN AND HILBERT SPACES
}

\author{
LUDVIK JANOS
}

(Received 8 June 1973)

Communicated by E. Strzelecki

\begin{abstract}
A subset $Y$ of a metric space $(X, \rho)$ is called rigid if all the distances $\rho\left(y_{1}, y_{2}\right)$ between points $y_{1}, y_{2} \in Y$ in $Y$ are mutually different. The main purpose of this paper is to prove the existence of dense rigid subsets of cardinality $c$ in Euclidean spaces $E_{n}$ and in the separable Hilbert space $l_{2}$. Some applications to abstract point set geometries are given and the connection with the theory of dimension is discussed.
\end{abstract}

\section{Introduction}

The concept of rigidity occurs in different branches of mathematics in different contexts. In topology it expresses the lack of non-trivial continuous mappings of a topological space into itself and it has a similar meaning in algebra. It appears natural to define rigidity of a metric space $(X, \rho)$ requiring the nonexistence of a nonidentical isometry $f: X \rightarrow X$ of $X$ onto itself. (See Janos (1972)). We adopt here a definition of rigidity which implies this condition, requiring that all the nonzero distances $\rho\left(x_{1}, x_{2}\right)$ in $X$ are mutually unequal which means that the distance function $\rho$ provides a one-to-one mapping $\left\{x_{1}, x_{2}\right\} \rightarrow(0, \infty)$ from the unordered pairs $\left\{x_{1}, x_{2}\right\}$ of points of $X$ into the interval $(0, \infty)$. If $(X, \rho)$ is a metric space, we introduce the concept of a rigid subset $T \subset X$ applying the above definition to the subspace $(Y, \rho)$.

\section{Rigid subsets in metric spaces}

DeFINITION 1.1. We say that a subset $Y \subset X$ of a metric space $(X, \rho)$ is rigid if $\rho\left(y_{1}, y_{2}\right)=\rho\left(y_{3}, y_{4}\right)$ and $y_{1} \neq y_{2}$ implies $\left\{y_{1}, y_{2}\right\}=\left\{y_{3}, y_{4}\right\}$ for all $y_{1}, y_{2}, y_{3}$, $y_{4} \in Y$. In particular any subset $Y$ of cardinality $|Y| \leqq 2$ less or equal 2 is rigid according to this definition.

REMARK 1.1. One may also define this property requiring that, given $a>0$ arbitrarily, there exists at most one solution $\left\{y_{1}, y_{2}\right\}$ in $Y$ of the equation $\rho\left(y_{1}, y_{2}\right)$ $=a$. The equivalence of both definitions is obvious. 
LEMMA 1.1. The family $\mathscr{R}$ of all rigid subsets of a metric space $(X, \rho)$ partially ordered by set-inclusion has a maximal element (is inductive).

PROOF. We check that the family is inductive; i.e., given any linearly ordered subfamily $\mathscr{R}_{1} \subset \mathscr{R}$ we show that $\cup \mathscr{R}_{1} \in \mathscr{R}$. Given $x_{1}, x_{2}, x_{3}, x_{4} \in \cup \mathscr{R}_{1}$ with $x_{1} \neq x_{2}$ and such that $\rho\left(x_{1}, x_{2}\right)=\rho\left(x_{3}, x_{4}\right)$ there is $Y \in \mathscr{R}_{1}$ such that $x_{1}, x_{2}, x_{3}$, $x_{4} \in Y$ implying that $\left\{x_{1}, x_{2}\right\}=\left\{x_{3}, x_{4}\right\}$ showing that $\cup \mathscr{R}_{1}$ is rigid.

DefINITION 1.2. Given $x \in X$ in a metric space $(X, \rho)$ and $r \geqq 0$ we denote by $S(x, r)$ the sphere about $x$ and of radius $r: S(x, r)=\{y \mid \rho(x, y)=r\}$. If $Y \subset X$ we denote by $\mathscr{D}(Y)$ the set of all nonzero distances in $Y: \mathscr{D}(Y)=\left\{\rho\left(y_{1}, y_{2}\right) \mid y_{1}\right.$, $y_{2} \in Y$ and $\left.y_{1} \neq y_{2}\right\}$. If $x_{1}, x_{2} \in X$ and $x_{1} \neq x_{2}$ we denote by $\left[x_{1}, x_{2}\right]$ their symetral: $\left[x_{1}, x_{2}\right]=\left\{y \mid \rho\left(x_{1}, y\right)=\rho\left(x_{2}, y\right)\right\}$. For any subset $Y \subset X$ we denote by $S(Y)$ and $[Y]$ the subsets of $X$ defined by:

$$
\begin{aligned}
S(Y) & =\bigcup\{S(y, r) \mid y \in Y \text { and } r \in \mathscr{D}(Y)\} \\
{[Y] } & =\bigcup\left\{\left[y_{1}, y_{2}\right] \mid y_{1}, y_{2} \in Y \text { and } y_{1} \neq y_{2}\right\} .
\end{aligned}
$$

Lemma 1.2. Let $Y \subset X$ be a rigid subset of a metric space $(X, \rho)$ and let $x \in X$ be a point in $X$ such that $x \notin Y$. Then the subset $Y \cup\{x\}$ is rigid if and only if $x \notin S(Y) \cup[Y]$.

Proof. Assuming $x \in S(Y) \cup[Y]$ we must show that $Y \cup\{x\}$ is no longer rigid. If $x \in S(Y)$ then there exists $y \in Y$ and $d \in \mathscr{D}(Y)$ such that $x \in S(y, d)$, but this implies that the equation $\rho\left(x_{1}, x_{2}\right)=d$ has at least two different solutions in $Y \cup\{x\}$. The one is namely $\left\{y_{1}, y_{2}\right\} \subset Y$ for which $\rho\left(y_{1}, y_{2}\right)=d$ and the other is $\{x, y\}$. (They are distinct since $x \notin Y$ ). If we assume $x \in[Y]$ then there exist $y_{1}, y_{2} \in Y$ such that $\rho\left(x, y_{1}\right)=\rho\left(x, y_{2}\right)$ so that the above equation has again at least two distinct solutions. Thus $Y \cup\{x\}$ is not rigid in this case.

Conversely, assuming that $Y \cup\{x\}$ is not rigid, the adjunction of the point $x$ to the rigid set violates this property in the sense that either there exists a distance $d=\rho\left(y_{1}, y_{2}\right)$ in $Y$ such that $\rho(x, y)=\rho\left(y_{1}, y_{2}\right)$ for some $y, y_{1} y_{2} \in Y$ and in this case we have $x \in S(y, d)$ or there exist $y_{1}, y_{2} \in Y$ such that $\rho\left(x, y_{1}\right)=\rho\left(x, y_{2}\right)$ and in this case we have $x \in\left[y_{1}, y_{2}\right]$. So in both cases we have $x \in S(Y) \cup[Y]$ which completes our proof.

DeFINITION 1.3. We say that a metric space $(X, \rho)$ is geometric, or has property $P_{1}$ if and only if all the spheres $S(x, r)(x \in X, r \geqq 0)$ and all the symetrals $[x, y](x, y \in X, x \neq y)$ in $X$ are nowhere dense in $X ;$ i.e., have no interior points in $X$.

DEFINITION 1.4. We say that a metric space $(X, \rho)$ has the rigidity developing property, or property $P_{2}$, if and only if given any finite rigid subset $Y \subset X$, any 
point $x \in X$ and any $\varepsilon$-neighbourhood $B(x, \varepsilon)$ about $x$, there exists a point $y \in B(x, \varepsilon)$ such that $Y \cup\{y\}$ is rigid.

REMARK 1.2. It is obvious that the finite metric space $(X, \rho)$ cannot have the property $P_{1}$, since the singletons $\{x\}$ are open in this case and they can be written in the form $S(x, 0)$. On the other hand, it can have the property $P_{2}$. This happens if and only if the whole space $(X, \rho)$ is rigid.

Lemma 1.3. The property $P_{1}$ implies the property $P_{2}$.

Proof. Let $Y$ be a finite rigid subset in $(X, \rho)$ with property $P_{1}$, let $x \in X$ and let $B(x, \varepsilon)$ be an $\varepsilon$-neighbourhood of $x$. Forming the set $S(Y) \cup[Y]$ we observe that since it is a finite union of closed sets without interior points it has empty interior. Hence the open set $B(x, \varepsilon)$ is not contained in $S(Y) \cup[Y]$ and therefore there is $y \in B(x, \varepsilon)$ such that $y \notin S(Y) \cup[Y]$. If $y \in Y$ then $Y \cup\{y\}=Y$ is rigid since $Y$ is rigid and if $y \notin Y$ then the rigidity of $Y \cup\{y\}$ follows from the Lemma 1.2 .

LeMmA 1.4. The property $P_{1}$ is hereditary with respect to dense subsets; i.e., given a metric space $(X, \rho)$ having the property $P_{1}$ and a dense subset $Y \subset X$ then the subspace $(Y, \rho)$ has again the property $P_{1}$.

Proof. We observe that if $U \subset X$ is open and $Y \subset X$ dense then the closure of $U \cap Y$ equals the closure of $U$. Given $y \in Y$ and $r \geqq 0$ the $r$-sphere about $y$ in $Y$ is the set $S(y, r) \cap Y$. Assuming that this set contains a nonempty open set in $Y$ there exists a nonempty open set $U$ in $X$ such that $U \cap Y \subset S(y, r) \cap Y$. Denoting by $\bar{A}$ the closure of a subset $A$ in $X$ we have:

$$
\bar{U}=\overline{U \cap Y} \subset \overline{S(y, r) \cap Y} \subset \overline{S(y, r)}=S(y, r)
$$

since the set $S(y, r)$ is closed. But this implies $U \subset S(y, r)$ contrary to the assumption that $(X, \rho)$ has property $P_{1}$. The same reasoning applies to the symetrals $\left[y_{1}, y_{2}\right] \cap Y$ in $Y$.

EXAMPLES. It is obvious that all Euclidean spaces $E_{n}(n=1,2, \cdots)$ with respect to the usual metric $\rho(x, y)=\sqrt{\sum_{k=1}^{n}\left(y_{k}-x_{k}\right)^{2}}$ have the property $P_{1}$ and hence also $P_{2}$. Using the last Lemma 1.4 we see that also the dense subspaces $\operatorname{Rat}\left(E_{n}\right) \subset E_{n}$ or Irrat $\left(E_{n}\right) \subset E_{n}$ (the set of points with all co-ordinates rationals or irrationals) also enjoy these properties. But it is not so obvious that these properties hold also for infinite dimensional linear spaces.

LEMMA 1.5. Any sphere $S(x, r)=\{y \mid\|y-x\|=r\}(x \in B, r \geqq 0)$ in a normed linear space $(B,\|\cdot\|)$ has empty interior.

PRoof. If $U \subset\{y \mid\|y-x\|=r\}$ were a nonempty open set in $B$, let $y \in U$ and consider the sequence $a_{k}=(1 / k) x+(1-1 / k) y(k=1,2, \cdots)$. Obviously is 
$a_{k} \rightarrow y$. Since $\left\|a_{k}-x\right\|=(1-1 / k)\|y-x\|=(1-1 / k) r$ it follows that for all $k a_{k} \notin S(x, r)$ contradicting to the assumption that $U$ is open in $B$ and containing $y$.

THEOREM 1.1. All Hilbert spaces (separable or not) have the property $P_{1}$.

ProOf. In view of the last lemma we have only to show that in any Hilbert space $(H,(\cdot, \cdot))$ the symetrals $\left[x_{1}, x_{2}\right]\left(x_{1} \neq x_{2}\right)$ have empty interiors. Using the translation $z \rightarrow z-\frac{1}{2}\left(x_{1}+x_{2}\right)$ we can without loss of generality assume that our symetral has the form $[-v, v]$ with $v \in H, v \neq 0$. But then we have $[-v, v]$ $=\{x \mid(x, v)=0\}$. If there were a nonempty open set $U$ in $[-v, v]$ with $x \in U$ then for some $\varepsilon>0$ all the vectors $x+y$ with $\|y\|<\varepsilon$ would belong to $[-v, v]$, hence $(x+y, v)=(y, v)=0$. Choosing $y$ parallel to $v$ we would reach the contradiction to our assumption $v \neq 0$, which completes our proof.

\section{Dense rigid subsets}

THEOREM 2.1. If $(X, \rho)$ is a separable metric space with the property $P_{2}$ then there is a dense rigid subset $Y$ in $X$.

Proof. In view of the Remark 1.2 we only have to deal with infinite spaces. Let $a, b \in X$ be two distinct points in $X$ and let $\left\{x_{n}\right\}$ be a dense sequence in $X$ (with repetitions or not). We set up a process constructing consecutively larger and larger rigid sets using repeatedly the property $P_{2}$ : choosing $\varepsilon=1$ and using $P_{2}$ there is a point $y_{1}{ }^{1} \in B\left(x_{1}, 1\right)$ such that the set $\left\{a, b, y_{1}{ }^{1}\right\}$ is rigid. Now choosing $\varepsilon=\frac{1}{2}$ and using $P_{2}$ again we construct rigid sets $\left\{a, b, y_{1}{ }^{1}, y_{1}{ }^{2}\right\}$ and $\left\{a, b, y_{1}{ }^{1}, y_{1}{ }^{2}, y_{2}{ }^{2}\right\}$ with $y_{1}{ }^{2} \in B\left(x_{1}, \frac{1}{2}\right)$ and $y_{2}{ }^{2} \in B\left(x_{2}, \frac{1}{2}\right)$. Continuing this way we construct rigid sets of the form $\left\{a, b, y_{1}{ }^{1}, y_{1}{ }^{2}, y_{2}{ }^{2}, \cdots, y_{1}{ }^{n}, y_{2}{ }^{n}, \cdots y_{n}{ }^{n}\right\}$ with $y_{1}{ }^{n} \in B\left(x_{1}, 1 / n\right), y_{2}{ }^{n} \in B\left(x_{2}, 1 / n\right) \cdots y_{n}{ }^{n} \in B\left(x_{n}, 1 / n\right)$. Defining $Y$ as the union of these sets it is obvious that $Y$ is rigid and also dense in $X$ as claimed.

Corollary. All Euclidean spaces $E_{n}$ and the separable Hilbert space $l_{2}$ possess dense rigid subsets.

Proof. It follows from Theorems 1.1. and 2.1 and from the fact that $P_{1} \rightarrow P_{2}$.

LeMMa 2.1. If a metric space $(X, \rho)$ possesses a dense rigid subset $Y \subset X$ then there is a maximal rigid subset $M \subset X$ in $X$ containing $Y$.

Proof. It follows from the inductive property of the family of all rigid subsets.

\section{Cardinality of maximal rigid subsets}

It is clear that the cardinality of any rigid subset $Y \subset X$ of any metric space $(X, \rho)$ cannot be larger than $c$, since the set of all distances $\mathscr{D}(Y)$ in $Y$ is in one-toone correspondence with the family of unordered pairs $\left\{y_{1}, y_{2}\right\} \subset Y$. Assuming the Continuum Hypothesis we will now show that in complete metric spaces with 
property $P_{1}$ every maximal rigid subset must have cardinality $c$. We will use the fact that a complete metric space has the Baire property (is of second category).

Theorem 3.1. Assuming Continuum Hypothesis every maximal rigid subset of a complete metric space $(X, \rho)$ with property $P_{1}$ has cardinality $c$.

Proof. The Baire Category theorem implies that $X$ cannot be countable since otherwise $X$ would be a countable union of singletons $\{x\}$ which are closed and nowhere dense since $\{x\}=S(x, 0)$ (Property $P_{1}$ ). Let $M \subset X$ be a maximal rigid subset of $X$. Using Continuum Hypothesis there are only two possibilities for $|M|$, namely either $M$ is countable or of cardinality $c$. Assume $M$ is countable, $M=\left\{y_{n}\right\}_{1}^{\infty}$, then the sets $S(M)$ and $[M]$ are of the first category as countable unions of nowhere dense sets $S\left(y_{n}, r_{m}\right)$ and $\left[y_{n}, y_{m}\right]$ respectively. On the other hand given $x \in X$ such that $x \notin M$ the Lemma 1.2 says that $x \in S(M) \cup[M]$ since $M \cup\{x\}$ cannot be rigid (maximality of $M$ ). Thus we obtain a representation of $X$ in the form: $X=M \cup S(M) \cup[M]$ implying that $X$ is of first category contrary to Baire Theorem, which completes the proof.

COROLLARY. Maximal rigid subsets of Euclidean spaces $E_{n}$ and of Hilbert spaces have cardinality $c$.

One may ask the question to what extent the size of maximal rigid subsets subsets $Y \subset X$ can be increased. For example: when is the cardinality of $Y$ larger than the cardinality of its complement $X \backslash Y$ ? We will show that in Euclidean spaces and in Hilbert spaces the cardinality of the complement $Y^{c}=X \backslash Y$ of any rigid subset $Y$ is always $c$.

LEMMA 3.1. Let $(X, \rho)$ be a metric space, $Y \subset X$ a rigid subset and $f: X \rightarrow X$ an isometric bijection of $X$ onto itself such that neither $f$ nor any of its powers $f^{n}$ has a fixed point in $X$. Then the intersection $Y \cap f(Y)$ is either empty or a one point set.

Proof. It is obvious that an isometric image of a rigid subset is again a rigid subset. Let us suppose that the intersection $Y \cap f(Y)$ is not empty. Thus there is $a \in Y$ such that $b=f(a) \in Y$. If $y \in Y$ is any element in $Y$ distinct from $a$ we consider the pair $\{a, y\}$ and its image $\{f(a), f(y)\}$. Since $\rho(a, y)=\rho(f(a), f(y))$ we conclude that either $\{a, y\}=\{f(a), f(y)\}$ or $f(y) \notin Y$. But the first case would imply $a=f(y)$ and $y=f(a)$ which in turn would imply $f^{2}(y)=y$ and $f^{2}(a)=a$ contrary to the assumption that no power of $f$ has a fixed point. Hence $f(y) \notin Y$ for all $y \in Y, y \neq a$ and we have in this case $Y \cap f(Y)=\{f(a)\}$ as claimed.

We are now ready to prove our theorem.

THEOREM 3.2. In the Euclidean spaces $E_{n}$ and in a Hilbert spaces (separable or not) the complements $Y^{c}$ of rigid subsets $Y$ have always cardinality $c$. 
Proof. In each of these spaces the translation $f: x \rightarrow x+a(a \neq 0)$ has the properties required in Lemma 3.1. Let $Y$ be a rigid subset. If $|Y|<c$ then of course we have $\left|Y^{c}\right|=c$. In case that $|Y|=c$ we use the result of Lemma 3.1. showing that either $f(Y) \cap Y=\theta \Rightarrow f(Y) \subset Y^{c}$ or $f(Y) \cap Y=\{b\} \Rightarrow f(Y) \backslash\{b\}$ $\subset Y^{c}$. Since $|f(Y)|=|Y|=c$ this implies that $\left|Y^{c}\right|=c$ as claimed.

\section{An application to abstract point set geometries}

An abstract point set geometry is a system $(\Sigma, \beta, A)$ where $\Sigma$ is a nonempty set of points, $\beta$ is a nonempty class of nonempty subsets of $\Sigma$ called blocks and $A$ is a list of axioms describing the meeting and covering done by blocks of $\beta$. (See Killgrove (1971).) We will give now a realization of one of these geometries where the set $\Sigma$ will be the underlying set of any Euclidean space $E_{n}$ or of an Hilbert space $H, \beta$ will be a certain subfamily of the family of all rigid subsets and the list of axioms $A$ will be: (using the notation adopted in Killgrove (1971))

$M_{2}$ If two distinct blocks meet their meet is a point.

$C_{3}$ For each pair of points $x, y$ there is at most one block $A$ containing both of them.

Let $H$ stand for any Euclidean or Hilbert space, and let $Y \subset H$ be any nonempty rigid subset in $H$. Let $G$ be the group of all translations of $H$. We define the family $\beta$ by: $\beta=\{g Y \mid g \in G\}$ and using Lemma 3.1 we observe that both axioms, $M_{2}$ and $C_{3}$ are satisfied. Our construction of a model for $(\Sigma, \beta, A)$, where $A=\left\{M_{2}, C_{3}\right\}$ depends on the choice of the rigid subset $Y$. If we choose $Y$ to have cardinality $c$ then our model enjoys the following property: $|\Sigma|=c,|\beta|=c$ (since $G$ has cardinality $c$ ), and finally each block $g Y \in \beta$ has cardinality $c$.

\section{Connection with the dimension theory}

In Janos (1972) it is proved that a separable metric space $(X, \rho)$ is zero-dimensional if and only if there exists a metric $\rho^{*}$ on $X$ which is topologically equivalent to $\rho$ and such that $\left(X, \rho^{*}\right)$ is rigid. The proof of this theorem is based on the following fact which we will need in the sequal:

LEMma 5.1. There is a metric $\rho$ on the Cantor set $C \subset[0,1]$ with the following properties:

(i) $\rho$ is topologically equivalent to the Euclidean metric in $C$,

(ii) $(C, \rho)$ is rigid,

(iii) for $x, y, z \in C, 0 \leqq x \leqq y \leqq z \leqq 1$ holds: $\rho(x, y)+\rho(y, z)=\rho(x, z)$.

For the proof see Janos (1972)

We will use this lemma to prove that rigid subsets of the real line $R$ form a universal model for separable zero-dimensional spaces in the sense that for any separable metrizable zero-dimensional space $X$ there exists a rigid subset $Y \subset R$ of the real line $R$ such that $Y$ is homeomorphic to $X$. We need the following lemma: 
LEMMA 5.2. There exists a rigid subset $C^{*} \subset R$ on the real line which is homeomorphic to the Cantor set $C$.

Proof. Using the metric $\rho$ on $C$ described in Lemma 5.1 we observe that the mapping $f: C \rightarrow R$ defined by $f(x)=\rho(0, x)$ for $x \in C$ is an isometry, since given $x, y \in C, x \leqq y$ we have, using the property (iii) of $\rho: \rho(0, x)+\rho(x, y)=\rho(0, y)$, thus $f(x)+\rho(x, y)=f(y)$ showing that $f(y)-f(x)=\rho(x, y)$. Defining $C^{*}$ as $f(C)$ it follows that $C^{*}$ is a rigid subset of $R$ and homeomorphic to $C$.

Now we are ready to prove the theorem:

THEOREM 5.1. Given any separable metrisable zero-dimensional space $X$ there is a rigid subset $Y \subset R$ of the real line which is homeomorphic to $X$.

Proof. It follows immediately from Lemma 5.2 and from the fact that every separable metrisable zero-dimensional space can be topologically embedded in the Cantor set.

This theorem shows a close relationship between zero-dimensionality and rigidity. A natural question arises whether this relationship can be extended and generalized to characterize $n$-dimensional spaces. It is well known that a separable metrisable space $X$ satisfies $\operatorname{dim}(X) \leqq n$ if and only if there exist $n+1$ dense zerodimensional subsets $Y_{1}, Y_{2}, \cdots, Y_{n+1}$ of $X$ such that $\bigcup_{1}^{n+1} Y_{k}=X$. So in view of the results so far obtained it seems reasonable to ask: Given a separable metrisable space $X$ with $\operatorname{dim}(X) \leqq n$. Does there exist a metric on $X$, compatible with the topology of $X$ and such that there are $n+1$ rigid subsets $Y_{1}, Y_{2}, \cdots, Y_{n+1}$ of $X$ such that $\bigcup_{1}^{n+1} Y_{k}=X$ ?

\section{References}

R. B. Killgrove (1971), 'Point set geometry', Math. Mag. 44, 115-119.

Ludvik Janos (1972), 'A metric characterization of zero-dimensional spaces', Proc. Amer. Math. Soc. 31, 268-270.

University of Newcastle Newcastle N.S.W. 2308

Australia 